Research Paper

\title{
Metastatic lymph node ratio as a prognostic indicator in patients with stage IV colon cancer undergoing resection
}

\author{
Chang Jiang $1,2,3^{*}$, Fang Wang ${ }^{*}$, Guifang Guo ${ }^{1,2,3}$, Jun Dong1,2,3,Shousheng Liu'1,2,3, Wenzhuo He ${ }^{1,2,3}$, Bei \\ Zhang1,2,3, Liangping Xia1,2,3凶 \\ 1. VIP Region, Sun Yat-Sen University Cancer Center, Guangzhou, Guangdong, 510060, R.P. China \\ 2. State Key Laboratory of Oncology in South China, Sun Yat-Sen University Cancer Center, Guangzhou, Guangdong, 510060, R.P. China \\ 3. Collaborative Innovation Center for Cancer Medicine, Guangzhou, Guangdong, 510060, R.P. China \\ 4. Department of Oncology, The First Affiliated Hospital of Sun Yat-sen University, Guangzhou, Guangdong, 510080, R.P. China. \\ * contributed equally to this paper \\ $\triangle$ Corresponding author: Liangping Xia, E-mail: xialiangpingsysucc@163.com, Fax: +86 20-87343392 Tel: +86 20-87343107 Mobile: +86-13926410608
}

(C) Ivyspring International Publisher. This is an open access article distributed under the terms of the Creative Commons Attribution (CC BY-NC) license (https://creativecommons.org/licenses/by-nc/4.0/). See http://ivyspring.com/terms for full terms and conditions.

Received: 2018.08.14; Accepted: 2019.05.05; Published: 2019.06.02

\begin{abstract}
Background: It has been shown that the metastatic lymph node ratio (LNR, metastatic LNs divided by the total number of retrieved LNs) significantly affects the prognosis of patients with non-stage IV and some curative stage IV colon cancer undergoing curative resection. In this study, we aimed to evaluate the role of the LNR as a predictor of prognosis in patients with stage IV colon cancer undergoing curative or palliative resection.

Patients and Methods: We conducted a retrospective study of 424 patients who were initially diagnosed with stage IV colon cancer at the Sun Yat-Sen University Cancer Center from 2003 to 2014. The patients were divided into the curative and palliative primary tumor resection groups with regional lymph nodes harvest. The median value was used as the cutoff for the LNR. Overall survival (OS) was assessed with the Kaplan-Meier method and log-rank test. Multivariate analysis was performed to identify the prognostic factors for OS.

Results: The cutoff value for the LNR was 0.2 . A total of 71 and 353 patients were classified as being in the curative and palliative resection groups, respectively. Patients in the palliative resection group showed higher pretreatment levels of carbohydrate antigen 19-9 (CA199; $P=0.014)$, a deeper infiltration of the primary tumor $(P=0.049)$, a lower regional lymph node harvest (i.e., total lymph node yield $[T L N] \leq 11$; $P=0.001)$, and more extensive metastasis $(P=0.006)$. Among all patients, initial elevated CA199 levels, a $T L N \leq 11$, a negative lymph nodes (NLN) $\leq 7$, and a $L N R \leq 0.2$ were significantly associated with an unfavorable prognosis. OS was significantly longer in patients with a low LNR in both groups $(P=0.008$ and $P=0.001$, respectively). The LNR was an independent prognostic indicator in patients with stage IV colon cancer, with a hazard ratio (HR) of $1.47(95 \%$ confidence interval $[\mathrm{Cl}] 1.14-1.91 ; P=0.003)$ in total population, and an HR of $1.43(95 \% \mathrm{Cl} 1.09-1.86 ; P=0.009)$ in patients with palliative resection.

Conclusion: The LNR can be used as an independent prognostic factor in patients with stage IV colon cancer patients undergoing resection.
\end{abstract}

Key words: lymph node ratio, colon cancer, prognosis, overall survival

\section{Introduction}

Colorectal cancer is one of the most common malignancies [1]. Approximately $15-25 \%$ of patients with colorectal cancer are initially diagnosed with synchronous metastases [2,3]. When the primary tumor and the metastatic lesions are resectable, curative surgery can improve the outcome [4]. The 
benefit of palliative resection of the primary tumor in patients with unresectable metastases remains unclear. Accumulating evidence indicates that certain populations can benefit from palliative resection [5-7]. In our previous study, we found that palliative resection improved overall survival (OS) and progression-free survival (PFS) in certain patients [8].

In colon cancer without distant metastasis, the post-surgical status of lymph nodes (LN) is a critical prognostic indicator, according to the International Union Against Cancer tumor-node-metastasis (AJCC/UICC TNM) grading system. Several other relevant indices, such as the total $\mathrm{LN}$ yield (i.e., the total number of LNs; TLN), the number of negative LNs (NLN), and the metastatic LN ratio (LNR), significantly affect the prognosis of patients with non-stage $V$ colon cancer[9] and other types of cancers such as breast cancer[10], lung cancer[11] and gastric cancer[12]. In patients with stage $\mathrm{V}$ colon cancer undergoing curative resection, an increased LNR was shown to be an independent predictor of decreased OS [13, 14]. In this study, we evaluated the significance of the metastatic LNR in patients with stage IV colon cancer who underwent curative or palliative resection of the primary tumor.

\section{Materials and Methods}

\section{Patients and treatment}

We retrospectively analyzed the complete data of 424 patients who were initially diagnosed with stage IV colon cancer at the Sun Yat-Sen University Cancer Center from January 2003 to December 2014. Patients who met the following criteria were enrolled: 1) underwent curative or palliative primary tumor resection with regional LN yield; the surgeries were either elective or emergency surgeries; 2) comprehensive assessment and multidisciplinary review were performed before the operation; 3) available records of routine blood tests before any treatment; 4) available paraffin-embedded tissue blocks; and 5) available follow-up information. Curative resection was defined as the complete resection of a macroscopic tumor (R0 resection) with negative pathological margins and no evidence of metastases [13].

Patients with rectal cancers, multiple colon cancers, hereditary colorectal cancer, inflammatory bowel disease, and second primary tumors as well as those who underwent an enterostomy were excluded from the study.

\section{Data collection}

Data on patient demographics, tumor characteristics, laboratory test results, and intact therapeutic procedures were obtained from our electronic medical records. The histopathology of the primary tumor, LN metastatic status, and perineural or vascular invasion status were confirmed by the Department of Pathology. Survival data were collected by telephone interview or review of medical records. The last follow-up was performed on September 30, 2017.

The LNR is defined as the number of metastatic LNs divided by the total number of retrieved LNs. Due to the lack of a uniform cutoff value, the median value of the LNR is generally accepted as the critical value $[15,16]$. In our data, a cutoff of 0.2 was used for the LNR. Similarly, the cutoff values for the TLN and NLN were 11 and 7 based on the median values, respectively. The upper normal values $(5 \mathrm{ng} / \mathrm{mL}$ and $35 \mathrm{ng} / \mathrm{mL}$ ) were used as the cutoff values for carcinoembryogenic antigen (CEA) and carbohydrate antigen 19-9 (CA199). OS was defined as the time from diagnosis to death for any reason or the last follow-up.

\section{Statistical analysis}

The distributions of the patient characteristics were assessed by the Chi square test. OS was estimated by the Kaplan-Meier method, and survival differences were analyzed by the log-rank test. Multivariate analyses and a Cox proportional hazards model were used to determine the independent prognostic factors for OS. SPSS version 23.0 (Statistical Product and Service Solutions, Chicago, IL, USA) was used to perform all statistical analyses. A $P$ value $<0.05$ was considered significant.

\section{Human Rights}

The protocol of our study was approved by the Ethics Committee of the Sun Yat-Sen University Cancer Center (GZR2015-034) and complied with the International Ethical Guidelines for Biomedical Research Involving Human Subjects, Good Clinical Practice guidelines, the Declaration of Helsinki, and local laws. Written informed consent was gathered from each patient.

\section{Results}

\section{Patient characteristics}

A total of 424 patients were enrolled in this retrospective study. Their baseline clinicopathologic features are shown in Table 1 . The median age at diagnosis was 58 years (range, 19-90 years), and $61.8 \%$ of the patients were men. Most patients (75.2\%) had initial elevated CEA levels, and $46 \%$ had increased initial CA199 levels. The primary lesion of 174 patients $(42.0 \%)$ located in the right colon. Only $17.5 \%$ of the patients had undergone treatment before the resection of the primary lesion, including 
A

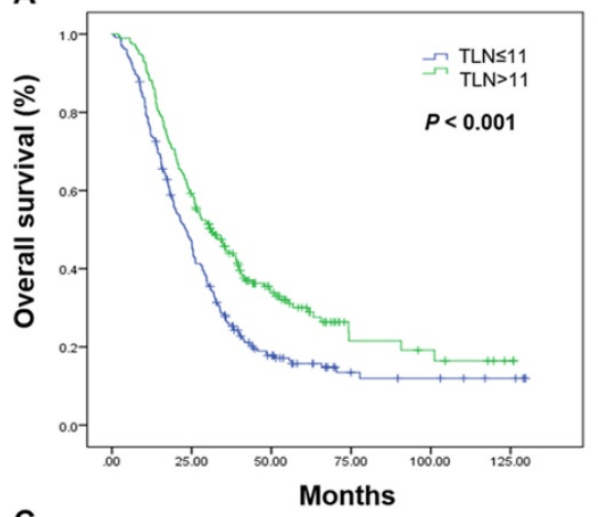

C

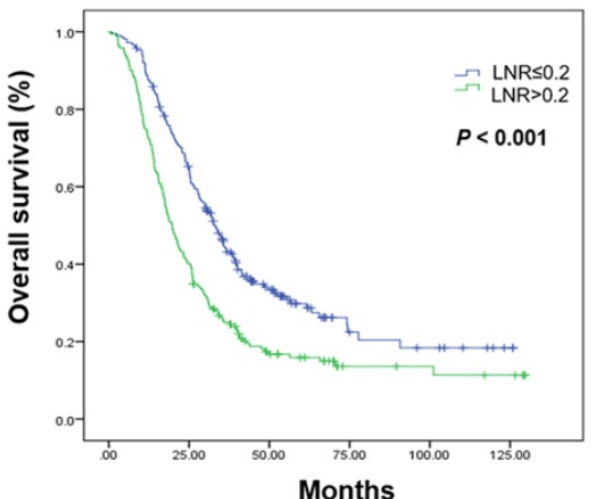

B

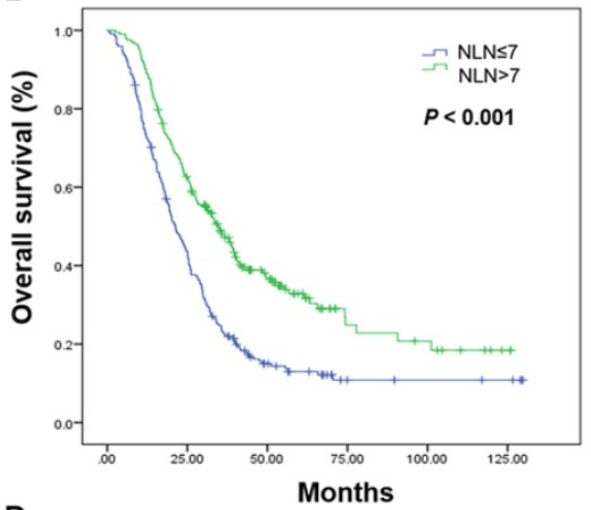

D

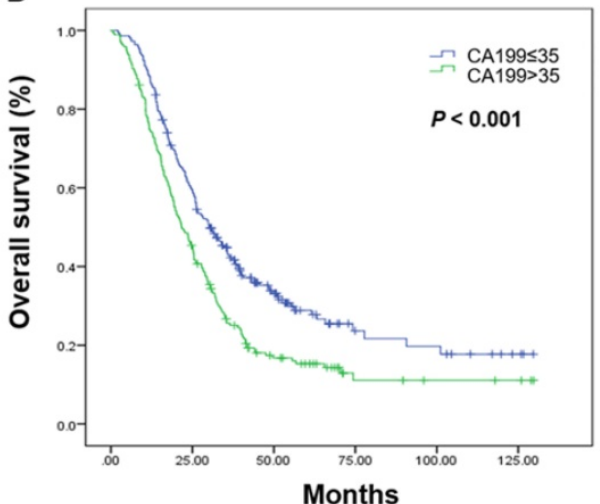

Figure 1. Kaplan-Meier estimates of overall survival. Overall survival was assessed in the total population according to the initial total lymph node yield (A), the number of negative lymph nodes (B), the metastatic lymph node ratio (C), and carbohydrate antigen 19-9 levels (D). Abbreviations: CA199, carbohydrate antigen 19-9; LNR, metastatic lymph node ratio; NLN, number of negative lymph nodes; OS, overall survival; TLN, totally lymph node yield

A

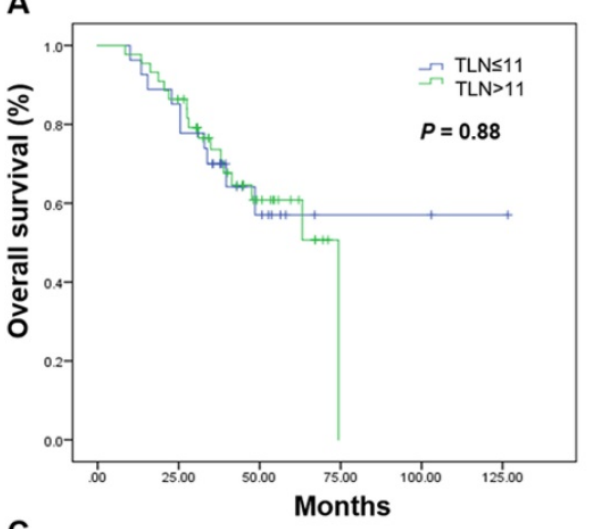

C

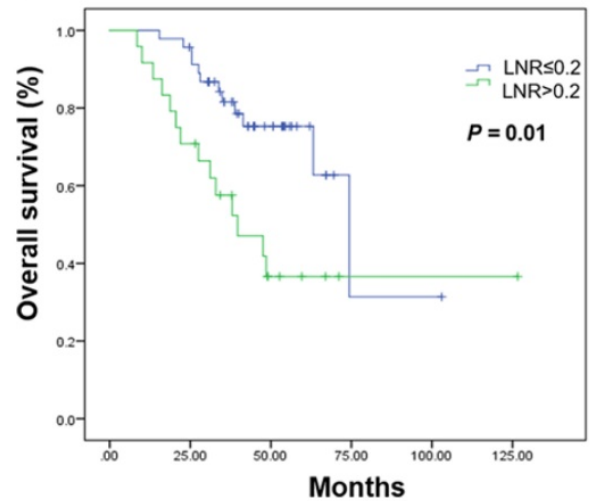

B



D

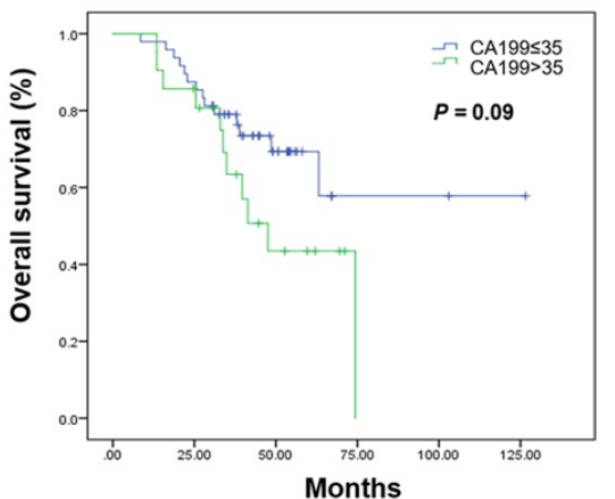

Figure 2. Kaplan-Meier estimates of overall survival in the curative resection group. Overall survival was assessed in the curative resection group according to the initial total lymph node yield (A), the number of negative lymph nodes (B), the metastatic lymph node ratio (C), and carbohydrate antigen 19-9 levels (D). Abbreviations: CA199, carbohydrate antigen 19-9; LNR, metastatic lymph node ratio; NLN, number of negative lymph nodes; OS, overall survival; TLN, totally lymph node yield 
Table 3. Overall survival according to lymph node status and serum carbohydrate antigen 19-9 levels by type of resection

\begin{tabular}{llll}
\hline & Curative resection group & & Palliative resection group \\
\hline & Median OS, months $(95 \% \mathrm{CI})$ & $P$ & Median OS, months (95\% CI) \\
\hline TLN $\leq 11$ & Not reached & 0.88 & $20.10(16.64-23.56)$ \\
$>11$ & Not reached & & $25.50(22.11-28.89)$ \\
NLN $\leq 7$ & $47.53(30.24-64.83)$ & 0.08 & $20.07(17.07-23.07)$ \\
$>7$ & $74.33(58.28-90.39)$ & & $26.00(20.35-31.66)$ \\
LNR 50.2 & $74.33(58.17-90.49)$ & 0.01 & $27.77(24.04-31.49)$ \\
$>0.2$ & $39.70(20.17-59.24)$ & 0.09 & $17.93(15.86-20.00)$ \\
CA199 $(\mathrm{ng} / \mathrm{mL})$ & & & 0.001 \\
$\leq 35$ & Not reached & & $25.60(23.16-28.04)$ \\
$>35$ & $47.53(33.65-61.42)$ & & $20.07(17.22-22.91)$ \\
\hline
\end{tabular}

Abbreviations: CA199, carbohydrate antigen 19-9; CI confidence interval; LNR, metastatic lymph node ratio; NLN, negative lymph nodes; OS, overall survival; TLN, total lymph node yield.

Table 4. Univariate and multivariate analyses of the factors affecting overall survival by Cox proportional hazard model

\begin{tabular}{|c|c|c|c|c|c|c|}
\hline \multirow[t]{2}{*}{ Variable } & \multicolumn{2}{|l|}{ Overall population } & \multicolumn{2}{|l|}{ Curative resection group } & \multicolumn{2}{|c|}{ Palliative resection group } \\
\hline & $\mathrm{HR}(95 \% \mathrm{CI})$ & $P$ & HR $(95 \% \mathrm{CI})$ & $P$ & HR $(95 \% \mathrm{CI})$ & $P$ \\
\hline \multicolumn{7}{|l|}{ Univariate analyses } \\
\hline Initial CA199 >35 & $1.43(1.16-1.77)$ & 0.001 & $1.30(0.06-2.83)$ & 0.51 & $1.31(1.05-1.63)$ & 0.016 \\
\hline TLN $<12$ & $0.65(0.52-0.82)$ & $<0.001$ & $1.06(0.48-2.34)$ & 0.88 & $0.69(0.54-0.87)$ & 0.002 \\
\hline $\mathrm{NLN} \leq 7$ & $0.55(0.44-0.68)$ & $<0.001$ & $0.51(0.23-1.09)$ & 0.82 & $0.65(0.51-0.82)$ & $<0.001$ \\
\hline $\mathrm{LNR} \leq 0.2$ & $1.72(1.37-2.15)$ & $<0.001$ & $2.74(1.27-5.93)$ & 0.011 & $1.51(1.19-1.92)$ & 0.001 \\
\hline \multicolumn{7}{|c|}{ Multivariate analyses } \\
\hline Initial CA199 >35 & $1.39(1.12-1.73)$ & 0.003 & $0.88(0.43-2.08)$ & 0.88 & $1.34(1.07-1.67)$ & 0.01 \\
\hline TLN $<12$ & $0.83(0.60-1.15)$ & 0.27 & $1.72(0.59-4.97)$ & 0.32 & $0.75(0.53-1.06)$ & 0.10 \\
\hline $\mathrm{NLN} \leq 7$ & $0.75(0.52-1.06)$ & 0.10 & $0.63(0.19-2.06)$ & 0.44 & $0.91(0.62-1.32)$ & 0.61 \\
\hline $\mathrm{LNR} \leq 0.2$ & $1.47(1.14-1.91)$ & 0.003 & $2.35(0.93-5.96)$ & 0.072 & $1.43(1.09-1.86)$ & 0.009 \\
\hline
\end{tabular}

Abbreviations: CA199, carbohydrate antigen 19-9; CI confidence interval; LNR, metastatic lymph node ratio; NLN, negative lymph nodes; OS, overall survival; TLN, total lymph node yield.

A

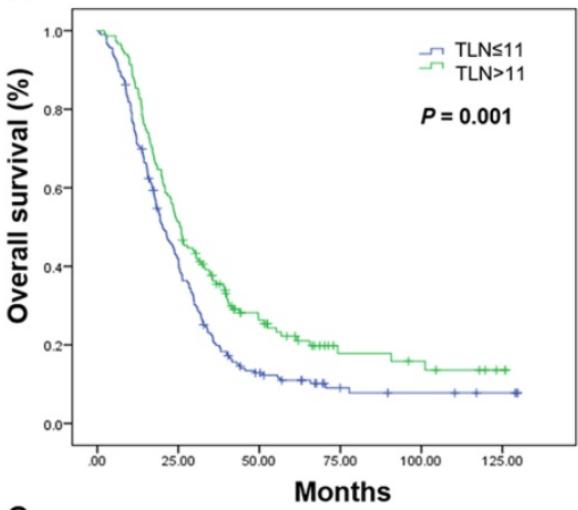

C

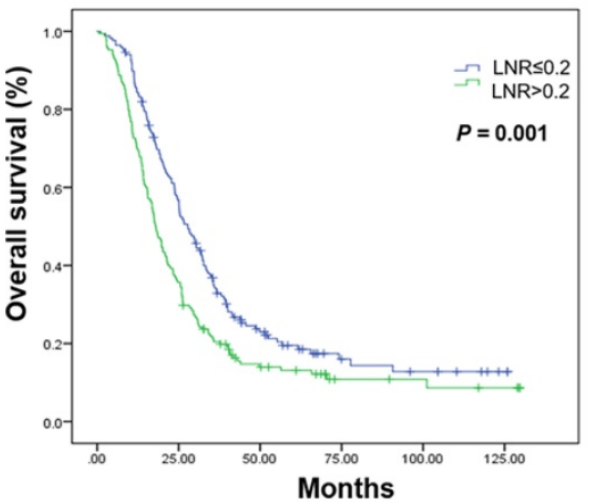

в
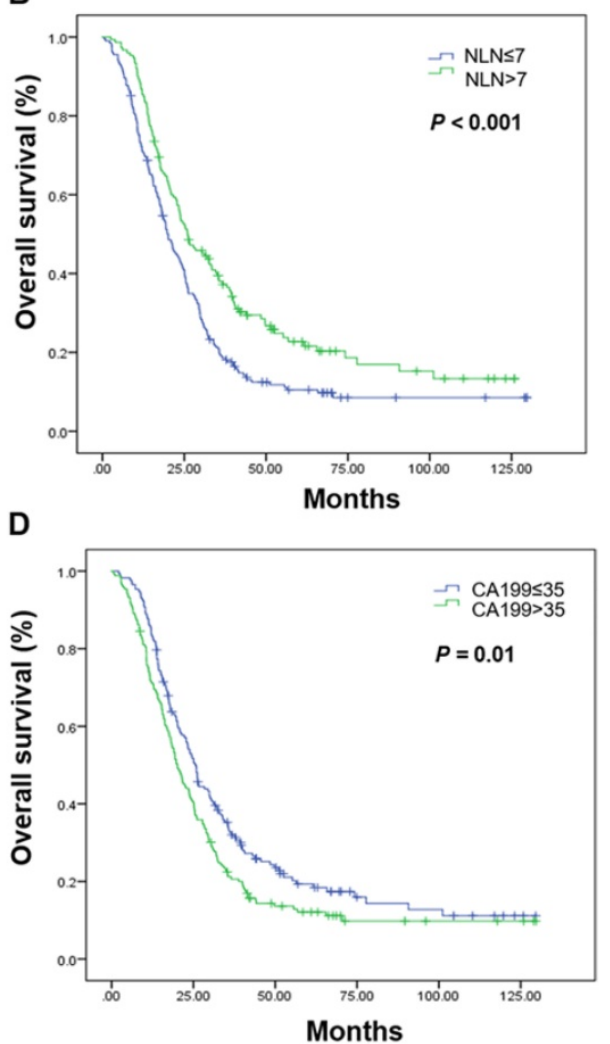

Figure 3. Kaplan-Meier estimates of overall survival in the palliative resection group. Overall survival was assessed in the palliative resection group according to the initial total lymph node yield (A), the number of negative lymph nodes (B), the metastatic lymph node ratio (C), and carbohydrate antigen 19-9 levels (D). Abbreviations: CA199, carbohydrate antigen 19-9; LNR, metastatic lymph node ratio; NLN, number of negative lymph nodes; OS, overall survival; TLN, totally lymph node yield 


\section{Discussion}

In the present study, we found the LNR can be used as an independent prognostic factor in patients with stage IV colon cancer who underwent curative or palliative resection of the primary tumor. Considering the heterogeneity of colon cancer, it is easy to understand that patients with a lower CA199 level, fewer metastases at multiple sites, and a less advanced tumor infiltration status were more likely to have a longer OS. Surgical removal of the primary tumor has been reported to improve the outcomes of potentially curable patients with colorectal cancer [17, 18]. The benefit of palliative resection in patients with unresectable metastases remains controversial. With the development of systemic chemotherapy and the treatment of local lesions, the benefit of palliative resection of the primary tumor in specific patient populations has been gradually recognized $[19,20]$. In our previous study, we found that patients with normal lactic dehydrogenase levels and a CEA level $<70 \mathrm{ng} / \mathrm{mL}$ could benefit from palliative operation surgery without serious surgery-related complications [8].

The prognostic factors of resectable metastatic colon cancer, such as the CEA level, tumor differentiation, and perineural invasion, have been extensively researched $[17,18,21]$. In this study, we found that the initial CA199 level was associated with OS in patients with resectable stage IV colon cancer. This is consistent with our identification of CA199 as an independent prognostic biomarker in patients with metastatic colorectal cancer [22].

The lymph nodes status is critical for prognosis in non-stage IV colon cancer in the current TNM staging system. Several studies have identified the LNR as a significant prognostic factor in patients with stage III colon cancer [23-25]; the LNR was a better prognostic factor that the positive LN count [16]. In stage IV colon cancer, researchers have suggested the prognostic significance of the LNR in patients with potentially curable disease [14, 26-28]. In patients with unresectable metastases, Ishihara et al. reported that negative LN dissection was associated with favorable outcomes [20]. Zhang et al reported more accuracy of survival prediction through the incorporation of negative LN into American Joint Committee on Cancer stages [29]. In our study, we further explored the role of the LNR in patients undergoing palliative resection. We identified the LNR as a in dependent negative predictor of prognosis in patients with metastatic colon cancer, specifically in those with unresectable metastases. A low LNR (high quota, rather than the number of retrieved LNs) could be interpreted as a strong anti-tumor immune response $[30,31]$.

The main limitations of this study are its retrospective study design and the relatively small number of patients with curative resection.

In conclusion, the LNR and initial CA199 levels were independently associated with OS in patients with metastatic colon cancer patients who underwent curative or palliative resection. Our data suggest that the LNR can serve as a prognostic indicator in patients with stage IV colon cancer and can be used to establish an adequate treatment strategy. Further validation studies are needed.

\section{Acknowledgements}

This study was supported by grants from the National Natural Science Foundation of China (81572409).

\section{Note}

Raw data was deposited in the Research Data Deposit system, http://www.researchdata.org.cn) of Sun Yat-Sen University Cancer and can be obtained from the corresponding authors on reasonable request.

\section{Competing Interests}

The authors have declared that no competing interest exists.

\section{References}

1. Msph LAT, Bray F, Siegel RL, Jacques Ferlay ME, Lortet-Tieulent J, PhD AJD. Global cancer statistics, 2012. Ca A Cancer Journal for Clinicians. 2015; 65: 87-108.

2. Nitzkorski J, Farma J, Watson J, Siripurapu V, Zhu F, Matteotti R, et al. Outcome and natural history of patients with stage IV colorectal cancer receiving chemotherapy without primary tumor resection. Ann Surg Oncol. 2012; 19: 379-83.

3. Scheer MG, Sloots CE, Gj VDW, Ruers TJ. Management of patients with asymptomatic colorectal cancer and synchronous irresectable metastases. Annals of Oncology Official Journal of the European Society for Medical Oncology. 2008; 19: 1829-35.

4. Lee WS, Baek JH, Kang JM, Choi S, Kwon KA. The outcome after stent placement or surgery as the initial treatment for obstructive primary tumor in patients with stage IV colon cancer. Am J Surg. 2012; 203: 715-9.

5. Bajwa A, Blunt N, Vyas S, Suliman I, Bridgewater J, Hochhauser D, et al. Primary tumour resection and survival in the palliative management of metastatic colorectal cancer tr. European Journal of Surgical Oncology. 2009; 35: 164-7.

6. Chan TW, Brown C, Ho CC, Gill S. Primary tumor resection in patients presenting with metastatic colorectal cancer: analysis of a provincial population-based cohort. Am J Clin Oncol. 2010; 33: 52-5.

7. Ahmed S, Leis A, Fields A, Chandrakanthan S, Haider K, Alvi R, et al. Survival impact of surgical resection of primary tumor in patients with stage IV colorectal cancer: results from a large population-based cohort study. Cancer. 2014; 120: 683-91.

8. He WZ, Rong YM, Jiang C, Liao FX, Yin CX, Guo GF, et al. Palliative primary tumor resection provides survival benefits for the patients with metastatic colorectal cancer and low circulating levels of dehydrogenase and carcinoembryonic antigen. Chin J Cancer. 2016; 35: 58.

9. Li Q, Liang L, Jia H, Li X, Xu Y, Zhu J, et al. Negative to positive lymph node ratio is a superior predictor than traditional lymph node status in stage III colorectal cancer. Oncotarget. 2016; 7: 72290-9.

10. Wang QX, Cai YF, Chen YY, Zhang W, Jin WX, Chen ED, et al. Additional Prognostic Value of Lymph Node Ratio (LNR) and Number of Negative Lymph Nodes (NLNs) in Chinese Patients with Triple Negative Breast Cancer. Ann Clin Lab Sci. 2017; 47: 68-75. 
11. Le DT, Durham JN, Smith KN, Wang H, Bartlett BR, Aulakh LK, et al. Mismatch repair deficiency predicts response of solid tumors to PD-1 blockade. Science. 2017; 357: 409-13.

12. Zhuo C, Ying M, Lin R, Wu X, Guan S, Yang C. Negative lymph node count is a significant prognostic factor in patient with stage IV gastric cancer after palliative gastrectomy. Oncotarget. 2017; 8: 71197-205.

13. Galizia G, Orditura M, Ferraraccio F, Castellano P, Pinto M, Zamboli A, et al. The lymph node ratio is a powerful prognostic factor of node-positive colon cancers undergoing potentially curative surgery. World J Surg. 2009; 33: 2704-13.

14. Ozawa T, Ishihara S, Nishikawa T, Tanaka T, Tanaka J, Kiyomatsu T, et al. Prognostic Significance of the Lymph Node Ratio in Stage IV Colorectal Cancer Patients who have Undergone Curative Resection. Ann Surg Oncol. 2015; 22: 1513-9.

15. Parnaby CN, Scott NW, Ramsay G, MacKay C, Samuel L, Murray GI, et al. Prognostic value of lymph node ratio and extramural vascular invasion on survival for patients undergoing curative colon cancer resection. Br J Cancer. 2015; 113: 212-9.

16. Ceelen W, Van Nieuwenhove Y, Pattyn P. Prognostic value of the lymph node ratio in stage III colorectal cancer: a systematic review. Ann Surg Oncol. 2010; 17: 2847-55.

17. Yun HR, Lee WY, Lee WS, Yong BC, Yun SH, Chun HK. The prognostic factors of stage IV colorectal cancer and assessment of proper treatment according to the patient's status. Int J Colorectal Dis. 2007; 22: 1301-10.

18. Stillwell AP, Ho YH, Veitch C. Systematic review of prognostic factors related to overall survival in patients with stage IV colorectal cancer and unresectable metastases. World J Surg. 2011; 35: 684-92.

19. Faron M, Bourredjem A, Pignon JP, Bouche O, Douillard JY, Adenis A, et al. Impact on survival of primary tumor resection in patients with colorectal cancer and unresectable metastasis: Pooled analysis of individual patients' data from four randomized trials. Plant Cell. 2012; 16: 3304-25.

20. Ishihara S, Nishikawa T, Tanaka T, Tanaka J, Kiyomatsu T, Kawai K, et al. Prognostic impact of tumor location in stage IV colon cancer: a propensity score analysis in a multicenter study. International Journal of Surgery. 2014; 12: $925-30$.

21. Venderbosch S, de Wilt JH, Teerenstra S, Loosveld OJ, van Bochove A, Sinnige $\mathrm{HA}$, et al. Prognostic value of resection of primary tumor in patients with stage IV colorectal cancer: retrospective analysis of two randomized studies and a review of the literature. Ann Surg Oncol. 2011; 18: 3252-60.

22. Jiang C, Liu S, He W, Zhang B, Xia L. The Prognostic and Predictive Value of Carbohydrate Antigen 19-9 in Metastatic Colorectal Cancer Patients with First Line Bevacizumab Containing Chemotherapy. J Cancer. 2017; 8: 1410-6.

23. Chen SL, Steele SR, Eberhardt J, Zhu K, Bilchik A, Stojadinovic A. Lymph node ratio as a quality and prognostic indicator in stage III colon cancer. Ann Surg. 2011; 253: 82-7.

24. Derwinger K, Carlsson G, Gustavsson B. A study of lymph node ratio as a prognostic marker in colon cancer. European Journal of Surgical Oncology the Journal of the European Society of Surgical Oncology \& the British Association of Surgical Oncology. 2008; 34: 771-5.

25. Berger AC, Sigurdson ER, LeVoyer T, Hanlon A, Mayer RJ, Macdonald JS, et al. Colon cancer survival is associated with decreasing ratio of metastatic to examined lymph nodes. J Clin Oncol. 2005; 23: 8706-12.

26. Derwinger K, Gustavsson B. A study of lymph node ratio in stage IV colorectal cancer. World Journal of Surgical Oncology,6,1(2008-12-01). 2008; 6: 1-6.

27. Galizia G, Orditura M, Ferraraccio F, Castellano P, Pinto M, Zamboli A, et al. The lymph node ratio is a powerful prognostic factor of node-positive colon cancers undergoing potentially curative surgery. World J Surg. 2009; 33: 2704-13.

28. Parnaby CN, Scott NW, Ramsay G, Mackay C, Samuel L, Murray GI, et al. Prognostic value of lymph node ratio and extramural vascular invasion on survival for patients undergoing curative colon cancer resection. Br J Cancer. 2015; 113: 212-9.

29. Zhang ZY, Gao W, Luo QF, Yin XW, Basnet S, Dai ZL, et al. A nomogram improves AJCC stages for colorectal cancers by introducing CEA, modified lymph node ratio and negative lymph node count. Sci Rep. 2016; 6: 39028.

30. Wong SL. Lymph node evaluation in colon cancer: assessing the link between quality indicators and quality. Jama the Journal of the American Medical Association. 2011; 306: 1139-41.

31. Park IJ, Choi GS, Jun SH. Nodal stage of stage III colon cancer: the impact of metastatic lymph node ratio. J Surg Oncol. 2009; 100: 240-3. 